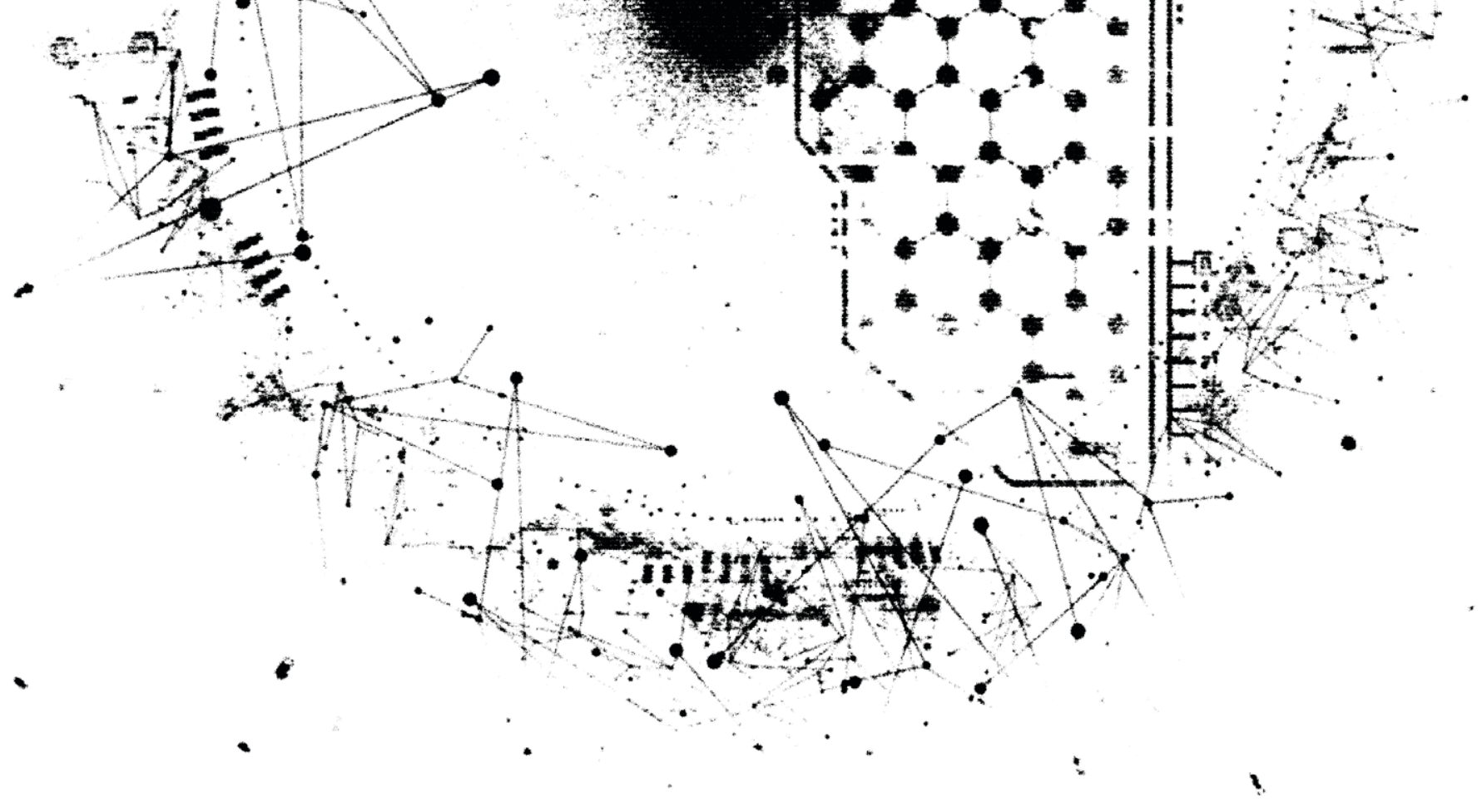

\title{
PERIODISMO DE DATOS EN LA TRANSICIÓN AL POSCONFLICTO EN COLOMBIA ${ }^{1}$
}

DATA JOURNALISM IN THE
POST-CONFLICT TRANSITION IN COLOMBIA

Por

Kevin Alexis García ${ }^{2}$

Universidad del Valle

kevin.alexis.garcia@correounivalle.edu.co

Orcid: http:// orcid.org/0000-0002-8412-9156

Julián González Mina ${ }^{3}$

Universidad del Valle

julian.gonzalez@correounivalle.edu.co

Orcid: http: / / orcid.org/0000-0002-0766-5772 
Resumen: Este artículo sustenta la hipótesis de que está emergiendo en Colombia un tipo especial de producción de periodismo de datos en el periodo de transición hacia el posconflicto armado. Mediante documentación bibliográfica y análisis exploratorio, se analizan seis proyectos de periodismo de datos, producidos por medios migrados y emergentes en internet que tienen como perspectiva central temáticas relacionadas con el conflicto. Se analiza en qué medida dichos proyectos entrañan una renovación y reconfiguración de las prácticas periodísticas usuales. El examen de los proyectos ha permitido identificar cuatro figuras de información a partir de los datos: 1. Procesos de geo y crono referenciación de eventos, 2. Visualización de contrastes y comparaciones, 3. Representación de vínculos de poder mediante grafos o redes, y 4. síntesis gráfica de información. A través del examen de los resultados se destacan los avances de estas iniciativas y se propone revisar el paradigma de interactividad en los medios. Se enfatiza que la débil exploración de la interactividad profunda offline/online es un desafío crucial para el desarrollo de medios de comunicación de nuevo y viejo tipo.

Palabras claves: Periodismo de Datos, Posconflicto, Colombia, Información, Interactividad.

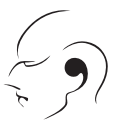

Abstract: This article supports the hypothesis that a special type of data journalism production is emerging in Colombia in the period of transition towards the armed post-conflict. Through bibliographic documentation and exploratory analysis, six data journalism projects are analyzed, produced by migrated and emerging media on the Internet, whose central perspective is related to the conflict. It is analyzed to what extent these projects involve a renewal and reconfiguration of the usual journalistic practices. The examination of the projects has allowed to identify four figures of information from the data: 1. Geo processes and chrono reference of events, 2. Visualization of contrasts and comparisons, 3. Representation of power links by graphs or networks, and 4. Graphic synthesis of information. Through the examination of the results, the advances of these initiatives stand out and it is proposed to revise the paradigm of interactivity in the media. It is emphasized that the weak exploration of deep offline / online interactivity is a crucial challenge for the development of new and old type media.

Keywords: Data Journalism, Post-Conflict, Colombia, Information, Interactivity. 


\section{Introducción}

Los conjuntos de datos a gran escala, también conocidos como macrodatos, su búsqueda, procesamiento, análisis e interpretación, vienen cobrando un valor creciente para dar sentido y producir valor en la era digital. Ferreras, en una aproximación al perfil profesional del periodista de datos (2013), sostiene que su trabajo es procesar el ingente volumen de datos a los que hoy tenemos acceso y convertirlos en piezas informativas comprensibles para los ciudadanos.

Frente a las tecnologías de la computación y el internet Manovich (2005) se propuso caracterizar el lenguaje de los nuevos medios e identificó cinco principios estructurales. Señaló que los medios en internet, aunque eran recientes, no eran nuevos desde una perspectiva histórica, si no ontológica. Es decir, estábamos ante medios de otra naturaleza y características; señaló como cinco de ellas: 1. La Representación numérica, 2. La modularidad, 3. La automatización, 4. La variabilidad y 5 . La transcodificación.

Con estos principios indicó que todo lo que veíamos y escuchábamos en internet: íconos, libros, voces, rostros, canciones, estaban compuestos de código digital, de representaciones numéricas, que podían ser sometidas a un manejo algorítmico y en consecuencia podían ser programables.

Señaló Manovich que la posibilidad de que los medios pudieran ser programables y modulares favorecía la automatización de sus procesos. Evidencia de ello es la existencia de programas que rastrean tendencias de los usuarios, parametrizan conductas para crear recomendaciones o descargan información de bases de datos públicas. Los principios anteriores favorecían a su vez la mutabilidad o variabilidad. Los medios en internet pueden cambiar con facilidad sus interfaces mediante plantillas que permiten modificar de manera instantánea su presentación pública. Sin embargo, la expresión más significativa de la variabilidad para el periodismo de datos era la escalabilidad; los medios, con procesos automáticos y programados podían manejar datos cambiando de forma significativa su volumen y escala.

Los principios anteriores ayudaron a comprender que internet era un medio pero también un poderoso metamedio, es decir, un manipulador y sintetizador de los medios que le precedieron y esta condición le permitía generar cambios con un alto nivel de aceleración.

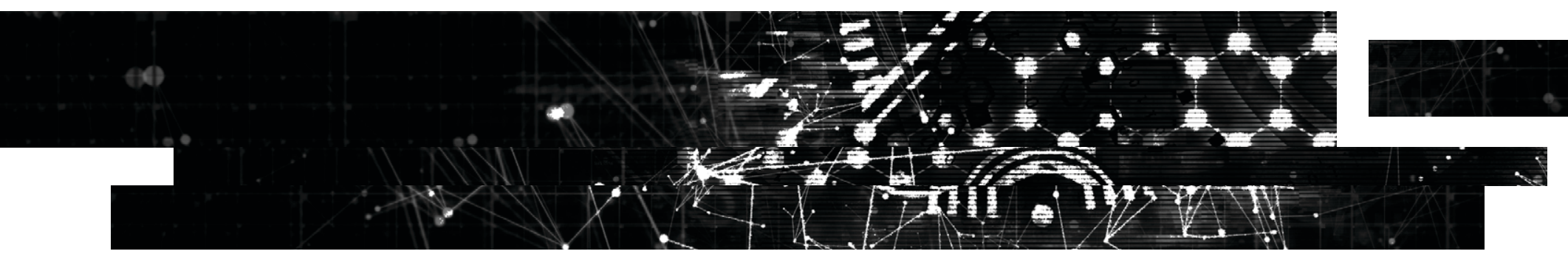


En el contexto latinoamericano Segnini (2008) introdujo el concepto de Periodismo Asistido por Computadora, el cual tenía estrechas conexiones y representaba una evolución del periodismo de investigación. Segnini definió este tipo de periodismo como cualquier proceso que utiliza la ayuda de las computadoras durante la recolección de insumos para las noticias. En el Periodismo Asistido por Computadora se cifraron las claves del periodismo de datos. Segnini asegura que el PAC no sustituye las técnicas de la reportería tradicional pero aprovecha las herramientas informáticas para lograr beneficios exponenciales y el acceso, administración y consecución de información.

Frente a investigadores tradicionales, que solían recolectar los directorios telefónicos de las ciudades que visitaban, con el periodismo asistido por computadora, un periodista podía obtener y almacenar voluminosa información en poco espacio y automatizar sus consultas. Para Segnini estos periodismos "multiplican las posibilidades para generar ideas para proyectos de investigación, minimizan la dependencia en las fuentes tradicionales y permiten al reportero obtener y entrevistar universos de datos para descubrir hechos ocultos" (2008, pág.46). El periodista puede cruzar variables entre bases de datos, obtener información precisa que puede ser verificada con el trabajo de campo y la reportería tradicional.

En 2006 Julián Assange registra el dominio Wikileaks, un proyecto definido como una agencia de inteligencia de la gente para filtrar los abusos del poder y solo tres años después tiene un millón doscientos mil documentos para su publicación. Assange aprovechó las posibilidades de internet 2.0, el cual potenció la automatización de procesos y permitió que los usuarios informáticos alrededor del mundo pudieran participar directamente en la producción de contenidos mediante el trabajo distribuido y colaborativo.

La emergencia en el escenario público de Assange supuso interrogantes sobre la necesidad de dar un manejo estructurado a grandes volúmenes de datos. En 2010 Tim Berners-Lee, el inventor de la World Wide Web sostiene en The Guardian que analizar los datos es el futuro de los periodistas ${ }^{4}$. Los periodistas debían intentar analizar los datos y equiparse con las herramientas necesarias para seleccionar lo interesante, mantener la perspectiva y ayudar a la gente a ver dónde encaja todo y qué sucede en su país.

Como han señalado Ferreras (2013) el periodismo de datos representa formas de evolución de técnicas de investigación que se fueron perfilando en Iberoamérica bajo el nombre de Periodismo Asistido Por Computadora y que se conocieron en los Estados Unidos como Computer-Assisted Journalism y ComputerAssisted Reporting.

Indican Gray, Bounegru \& Chambers (2012) que la reportería asistida por computador fue usada por primera vez en 1952 por parte de la CBS para predecir los resultados de la elección presidencial. Indican que desde esa época los periodistas han procurado ejercer control sobre el poder de forma independiente mediante el manejo de bases de datos de registros públicos.

Esta corriente del periodismo está emparentada con los orígenes del Periodismo de precisión, utilizado por primera vez por Everette Dennis en Estados Unidos (Galindo, 2004); se destacaron bajo esta última categoría piezas periodísticas que incorporaban adaptaciones de las técnicas de investigación de las ciencias sociales. Pero sería Philip Meyer en 1973 quien instituiría el concepto a través de Precision Journalism, un libro que marcaba diferencias contra la corriente subjetivista del llamado Nuevo Periodismo norteamericano. 
Para Holovaty una barrera al cambio se presentaba en que los periodistas estaban más preocupados por delimitar qué era y que no era periodismo y debían centrarse más en producir información útil para la vida de las personas y ayudarles a comprender el mundo. A través de las bases de datos los medios pueden permitir que las audiencias puedan explorar los hechos crudos de la historia, para encontrar detalles, hacer comparaciones, identificar jerarquías, prevalencias y múltiples valores.

Juanita León (2011), fundadora del portal lasillavacia.com/, un medio pionero en periodismo de datos en la zona latinoamericana, en su artículo Eight Ways the Internet Changes the Way We Think about the News (Ocho maneras en que internet cambia la forma en que pensamos las noticias), señalaba en 2011 en una publicación digital de la Universidad de Harvard que el concepto de noticia tal como se conocía en el periodismo tradicional había mutado en internet.Y señala ocho aspectos distintivos. Para León hoy es historia periodística 1. La información cruda, 2. El contexto, 3. El proceso de hacer la historia, 4. La construcción colectiva de la noticia, 5. El cubrimiento en tiempo real, 6. Las experiencias de inmersión virtual, 7. Las herramientas y la 8. La conversación agregada y comentada por los usuarios.

Para León, se trata entonces de aprovechar el proceso de construcción y convertirlo en historia misma, de posibilitar una participación activa de las audiencias en la forma de diseñar y perfilar los trabajos, de aprovechar creativamente los desarrollos tecnológicos, como el streaming y la realidad virtual inmersiva, entendidos como agentes que posibilitan nuevas posibilidades sensibles y espacio-temporales para comprender los temas. La suya es una propuesta que de forma deliberada crea porosidades, difumina las fronteras en los procesos de producción periodística y entiende los medios como instancias abiertas, más transparentes y participativas.

Bounegru, Gray \& Chambers (2012), en el Manual de Periodismo de Datos señalan que hoy las noticias fluyen en el mismo tiempo en que están sucediendo, se diseminan a través de múltiples fuentes, creando una red de conexiones que no siempre es comprensible como conjunto. De allí el valor del periodismo por recuperar los hechos aislados y dirimir los patrones y tendencias de las prácticas sociales. Esto como un mecanismo para hacer explicable la complejidad social. Los periodistas han requerido acceder a los datos crudos y disminuir su dependencia al manejo de los datos de las fuentes oficiales.

Por su parte, Ávila (s.f), en el libro en línea Manual de periodismo de datos iberoamericano, sostiene que poder extraer gran cantidad de información y sistematizarla no debe considerarse aún periodismo de datos. Para ella, acceder a las bases de datos no es el fin en sí mismo. Se trata entonces de centrarse en el interés público para encontrar las asociaciones que pasaban inadvertidas y aprovechar las obligaciones de los gobiernos de transparentar sus actividades para fortalecer la vigilancia al poder ${ }^{6}$. 


\section{Panorama del periodismo de datos}

En su investigación sobre el periodismo de datos en España, Ferreras (2016) ya anticipa un panorama preliminar de unidades de datos en medios importantes a nivel global. Es progresivo el interés de los medios de gran incidencia en la opinión pública: En Estados Unidos The New York Times, con su sección The Upshot, The Washintong Post, con Data Post y Los Angeles Times, con Data Desk. También es destacado el papel de medios emergentes constituidos por equipos con periodistas de alta trayectoria que migraron de los medios tradicionales y periodistas jóvenes y profesionales de las ingenierías y el diseño.

El trabajo más significativo lo ha logrado Propublica, un medio nativo digital, nacido en 2007 que cuenta con un equipo de más de 75 periodistas dedicados y opera como una sala de redacción independiente. Una de las claves de Propublica es que ha entendido la condición de internet y ha operado como medio que genera contenido directo para sus audiencias, y como metamedio proveedor de otros medios, capaz de establecer convenios con informativos tradicionales, proveer historias y lograr publicaciones conjuntas.

En Europa ha sido destacado la unidad de datos de The Guardian llamada Datablog, en Alemania Zeit Online con su sección Datenjournalismus y en Italia se ha destacado Dataninja. En el contexto europeo también un proyecto notable es Investigative Dashboard. Esta plataforma ofrece apoyo gratuito a reporteros de cualquier parte del mundo para rastrear personas, empresas y activos, y dispone un robusto directorio de bases de datos públicas alrededor del mundo.

Estos medios han logrado investigaciones notables en sus países mediante la creación y visualización de bases de datos. Sin embargo, el proyecto de mayor impacto global lo ha alcanzado ICIJ, el Consorcio Internacional de Periodistas de Investigación (por sus siglas en inglés $)^{7}$. Una red global de más de 200 periodistas de investigación y 100 organizaciones de medios en 70 países que desarrollan proyectos colaborativos para producir historias de profundidad. ICIJ produjo el mayor proyecto de periodismo de datos de impacto global. Si Wikileaks filtró 1,2 millones de documentos, en The Panama Papers los periodistas colaboradores de ICIJ procesaron 11,5 millones de documentos, de 40 años de operaciones de Mossack Fonseca, un despacho de abogados con sede principal en Panamá. El análisis de los datos reveló información sobre más de 214.000 compañías conectadas con personas en más de 200 países $^{8}$. Este trabajo permitió exponer la forma en que las compañías alrededor del mundo aprovechan los paraísos fiscales para lavar sus activos mediante compañías secretas offshore.

En los países de América Latina se ha ido consolidando una producción de periodismo de datos que ha logrado un reconocimiento de impacto global. Han sido elegidos ganadores por sus proyectos en los Data Journalism Adwards los medios La Nación 
de Argentina por su proyecto Declaraciones Juradas Abiertas, una base de datos que permitía visualizar las cifras de 260 funcionarios con información pública sobre sus propiedades y patrimonios. También fueron ganadores los proyectos peruanos de Ojo Público Cuentas juradas: un análisis de los cambios y la riqueza de los alcaldes de Lima y Excesos sin castigo, un proyecto de minería de datos del medio Convoca que siguió el rastro ambiental de las industrias

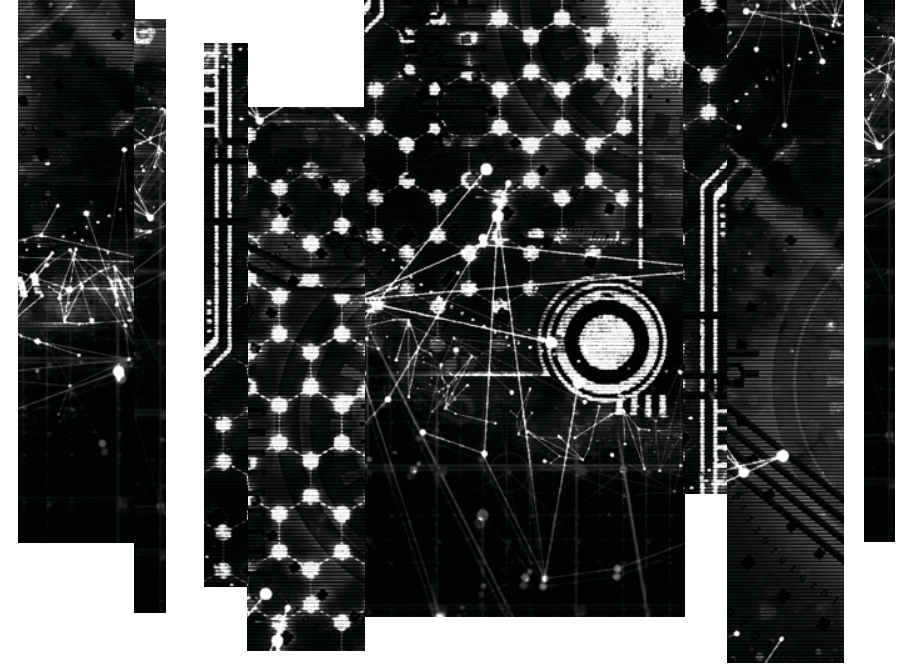
extractivas en Perú. Por último, también recibió reconocimiento global el proyecto Rutas del Conflicto, creado por el medio Verdad Abierta (s.f.). Una iniciativa que facilita el acceso a la información sobre el conflicto armado colombiano.

Respecto al país de estudio, en Colombia de forma paulatina los medios nacionales y regionales han ido incorporando producción de periodismo de datos: El Tiempo, El Espectador, El País, y Vanguardia entre los medios migrados han logrado generar contenidos; y a su vez han emergido proyectos nativos de internet destacándose entre estos La Silla Vacía. En Colombia la organización Consejo de Redacción, presidida por Gina Morelo, editora de la Unidad de Datos de El Tiempo, ha liderado el proceso de formación en está línea.

\section{El conflicto armado en Colombia}

Durante la segunda mitad del siglo XX Colombia vivió un periodo de violencia sostenida y sistemática que se fue transformando con el paso de las décadas y en la que se enfrentaron fuerzas del Estado, grupos guerrilleros, grupos paramilitares, carteles del narcotráfico y bandas criminales. En 2013, en medio de diálogos entre la guerrilla de las Farc y el gobierno colombiano, el Centro Nacional de Memoria Histórica, una institución creada por el Estado para esclarecer el conflicto armado en el país, presentó el informe “¡Basta Ya! Colombia: memorias de guerra y dignidad” (2013). Los investigadores señalaron que entre 1958 y 2012 el conflicto armado causó la muerte a más 218.000 personas, de las cuales 177.000 pertenecían a la población civil. En un periodo de cuarenta años (1970-2010) más de 27 mil personas fueron secuestradas, el 90\% de los secuestros fue cometido por las guerrillas. También se presentaron más de 10 mil víctimas por minas que dejaron 8 mil lesionados.

En medio de la degradación de la guerra, el conflicto se escaló en el inicio de los años ochenta con la emergencia de los grupos narcotraficantes, la violencia sicarial y paramilitar. Según el informe, entre 1985 y 2012, se presentaron 1.982 masacres, que dejaron más de 11 mil víctimas; más de la mitad de estas masacres fueron cometidas por grupos paramilitares, mientras 15 municipios registraban estados críticos y susceptibles a los asesinatos colectivos. 
Entre 1985 y 2012 se presentaron más de 25 mil víctimas de desapariciones forzadas y más de 5.700 .000 personas fueron desplazadas. En medio de ese contexto, con más de cincuenta años de conflicto armado, el informe ¡Basta ya! señalaba la magnitud, ferocidad y degradación de la guerra. Indicaba que el carácter invasivo de la violencia y su larga duración han impedido que se reconozca a los actores del conflicto y sus lógicas, así como a las víctimas.

En medio de la polarización por tensiones entre los partidos políticos de derecha y ultraderecha en el poder, en septiembre de 2016 el gobierno colombiano y las Farc firmaron el acuerdo de Paz. En la actualidad Colombia se ve avocado a esclarecer las dimensiones de su propia tragedia. Se asiste a un periodo de transición y en dicho periodo generar un cambio social implica garantizar por parte de los actores históricamente enfrentados los derechos a la verdad, la justicia, la reparación, la garantía de no repetición y la construcción de una paz que comprometa a varias generaciones de colombianos.

En un contexto de alta complejidad los archivos físicos y digitales favorecen la construcción de la memoria histórica, las bases de datos que registran hechos victimizantes operan como sistemas de vigilancia a la implementación de los acuerdos y el cumplimiento de los puntos pactados. En este periodo se requiere un manejo estructurado de datos que favorezcan los procesos de justicia, reparación y distribución de responsabilidades, así como el reconocimiento de los hechos por parte de los victimarios; procesos que permitan la identificación de las víctimas para sus procedimientos de atención, asistencia y reparación; datos que permitan el esclarecimiento de los hechos de violencia ocurridos durante el conflicto y ayuden a orientar la búsqueda de personas desaparecidas.

Este artículo sustenta como hipótesis que se está produciendo en Colombia un fenómeno emergente de producción de periodismo de datos en el periodo de transición hacia el posconflicto armado. Frente a un conflicto de más de medio siglo, con varios millones de desplazados, proyectos especiales sobre desaparecidos, violencia urbana en las capitales, cronología de masacres por actores armados, caracterización de mapas de poder y cartografías regionales buscan contribuir a la comprensión de la complejidad de nuestras violencias.

\section{Material y método}

Se analiza un corpus de piezas periodísticas colombianas basadas en periodismo de datos, referidas al conflicto político y armado colombiano, publicadas en medios digitales, algunos de ellos claramente emergentes (García, 2016). Se examinan los proyectos Rutas del conflicto: masacres ocurridas en Colombia desde el año 1982 hasta 20139", Colombia en Desaparecidos Duelo Eterno (ElTiempo, 2015) ${ }^{10}$, El mapa de la muerte ${ }^{11}$ (El País, 2016), Paz en Terreno ${ }^{12}$ (Rutas del Conflicto, El Espectados, ICFJ, Friederich Ebert Stiftung, s.f.), El Magdalena medio intenta cicatrizar sus heridas ${ }^{13}$ (Verdad Abierta, 
2016) y Quién es Quién ${ }^{14}$ (La Silla Vacía, 2016). Se busca comprender en qué sentido estamos ante formas del ejercicio periodístico que no sólo están haciendo una importante contribución al esclarecimiento y comprensión de nuestro prolongado conflicto armado, político y social de cinco décadas, sino también entrañan una auténtica renovación y reconfiguración de nuestras prácticas periodísticas usuales.

\section{RUTAS
DEL
CONFLIOTO}

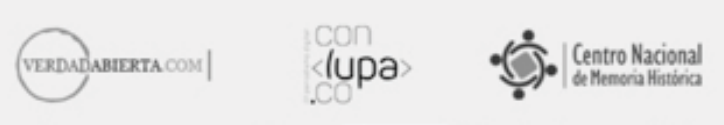

\begin{tabular}{llll}
\hline If Compartir $5,3 \mathrm{mil}$ & Tweet Google + & 1
\end{tabular}

\section{INICIO | ELPROYECTO I DESTACADOS । CARTOGRAF́́A DEL CONFLICTO | YOSOBREVIVÍ । MULTIMEDIA}

\section{Descarga la aplicacion \\ en tu smart Phene. \\ Aapwancen en \\ App Store \\ Google play \\ CARTOGRAFÍA DEL CONFLICTO}

REALIZA TU BÚSQUEDA >

BUSCAR

Este mapa muestra la cartografía de las masacres ocurridas en Colombia desde el año 1982 hasta 2013. Seleccione el año sobre el que desea ver el número de masacres

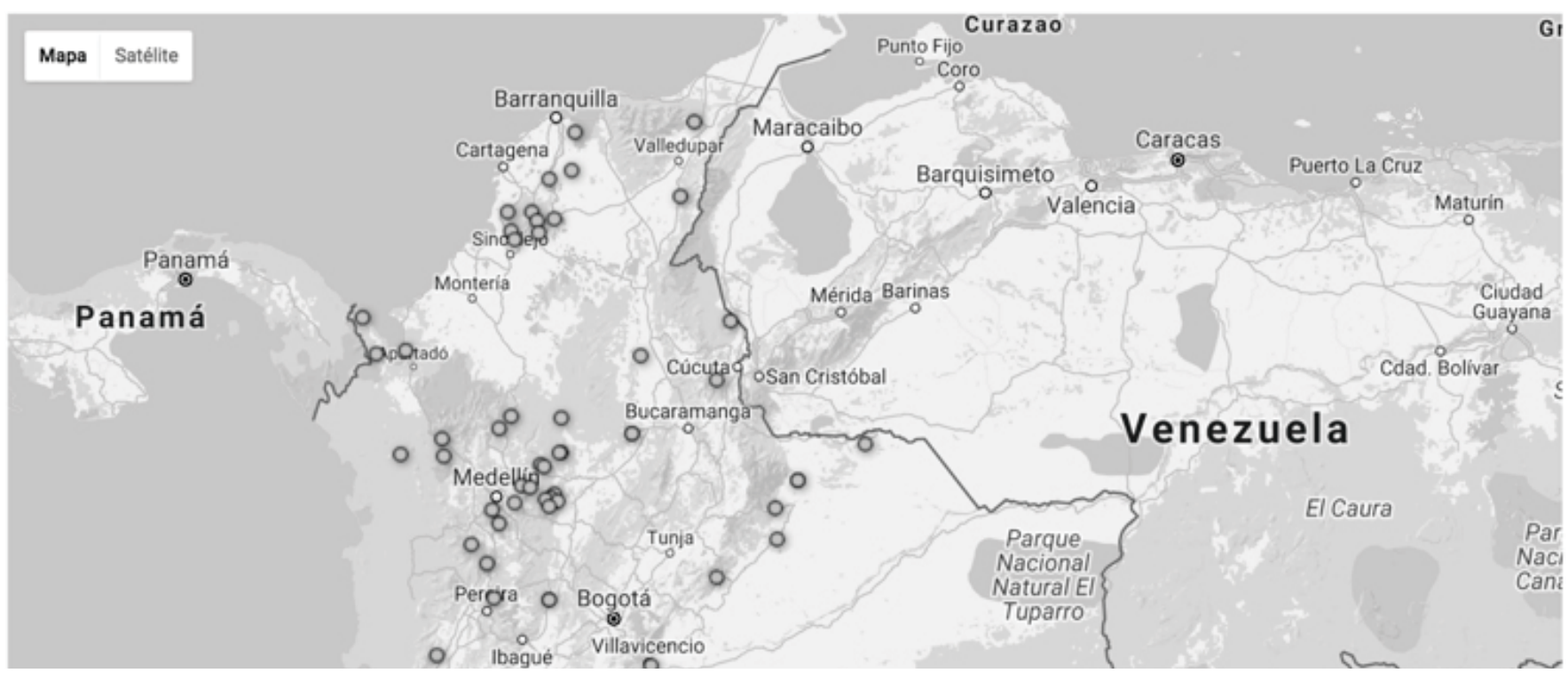

Figura 1. Proyecto Rutas del Conflicto.

http://rutasdelconflicto.com/geografia-del-terror/masacres_desaparecidos.php

En ocasiones los hábitos mentales nos procuran atajos que nos ahorran trabajo innecesario. Pero con frecuencia esos atajos nos impiden ver los aspectos claves de un fenómeno, como cuando buscamos en todos lados las gafas perdidas que tenemos atadas a nuestras narices. Habituados a hablar de datos e información, damos por descontado y sobreentendido de qué hablamos cuando lo hacemos. 
¿Qué es exactamente un dato? O, mejor, ¿cuándo ocurre que un recurso de información o dato se convierte en información? Flores (1994) distingue entre dato e información: "La segunda verdad es que la información no eran los data, sino que ese cambio en los datos era el que producía una diferencia, y que, en el concepto de información, el tiempo jugaba un papel importante. Data en momentos distintos podían producir gran valor, o perder valor totalmente; es por eso que, para nosotros, leer el diario de ayer, no interesa tanto como el de hoy por la mañana" (Flores, 1994, pág. 16). En su artículo seminal y clásico, Shannon (1948) también subraya cómo información es el diferencial, y no el recurso en sí mismo. Y en un campo completamente diferente, el de la psicología de la percepción, Gibson (1972/2002) entiende de manera relacional, ecológica y situada la información perceptual como variación experimentada por un sistema vivo. Si información está relacionada con valor diferencial y tiempo, ¿en qué momento los datos del periodismo de datos se convierten en información?

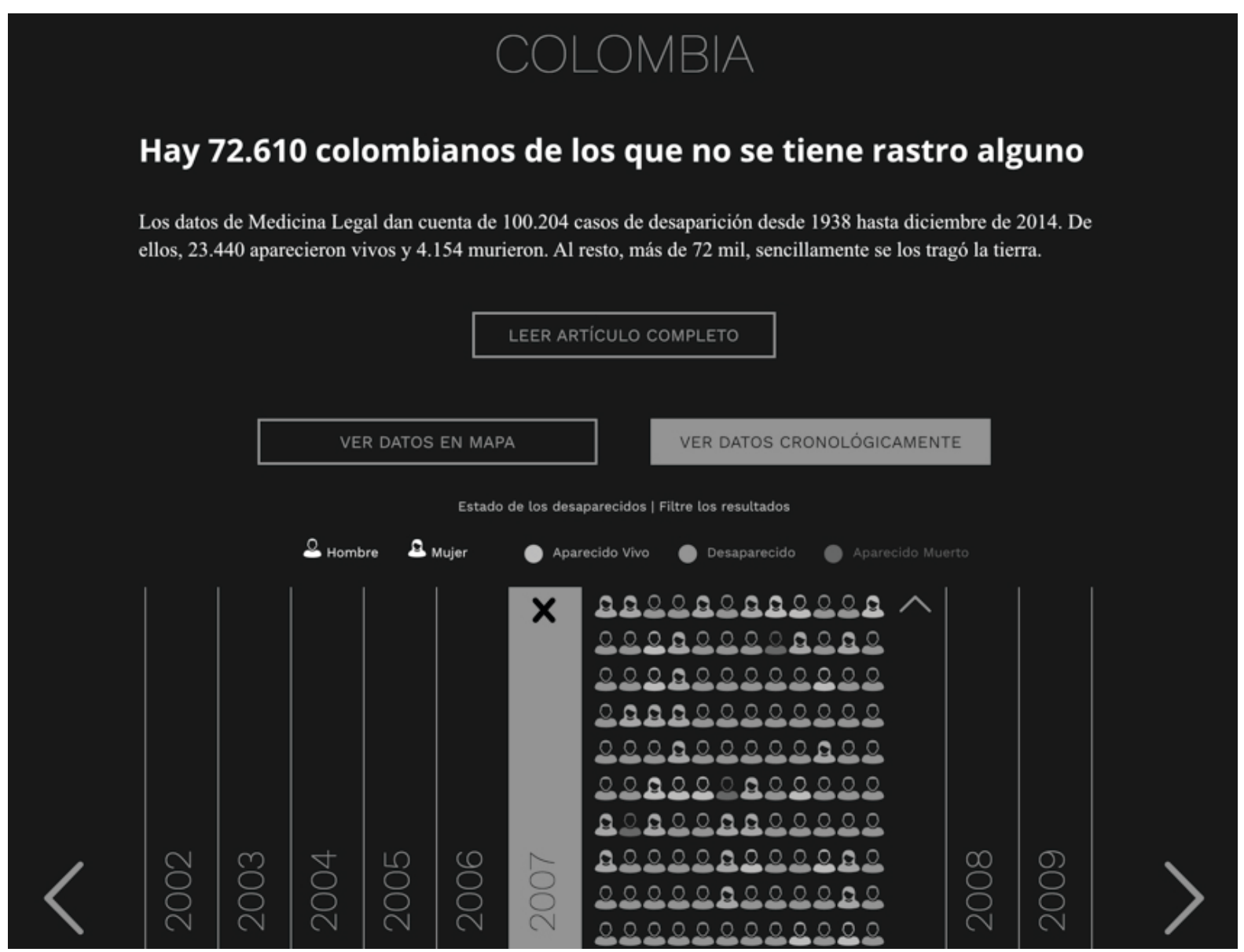

Figura 2. Proyecto Desaparecidos: Duelo eterno.

http://www.eltiempo.com/multimedia/especiales/desaparecidos-duelo-eterno/16382245/1/index.html 
Habría tres momentos en que los datos o recursos de información se transforman en valor diferencial: cuando el sistema A organiza tales recursos y les proporciona una nueva configuración al establecer relaciones nuevas entre los recursos previos o data anteriores; cuando el sistema B usa esos recursos o data nuevos producidos por el sistema A, para -en el tiempo T- proceder, actuar y comprender; y cuando los propios recursos cambian en el tiempo ${ }^{15}$. El periodismo de datos, como práctica de producción de comunicación pública, toma recursos o datos disponibles y los reorganiza, vincula y procesa configurando un nuevo recurso. También lo hacen las prácticas periodísticas convencionales, que transforman recursos obtenidos mediante inspecciones, conversaciones, entrevistas e inferencias en nuevos recursos. Lo que define específicamente al periodismo de datos es que estos procesos se despliegan en entornos de nuevos medios, lo que supone un sustancial aumento de la potencia de archivado, la ampliación de procesos automáticos, oportunidades de programación a partir de variaciones del sistema, rica y abundante presencia de hipervínculos, rutas diversas de uso y navegación (Manovich, 2005) y la amigabilidad que procuran los software de medios (Manovich, 2013).

Pero el dato o recurso de información, reorganizado y transfigurado, no constituye el proceso completo de comunicación pública. Requiere su transformación en sentido mediante el uso inteligible e inteligente de otro agente o sistema, en este caso el usuario. La literatura sobre periodismo de datos suele enfatizar el punto de vista del agente productor, en los nuevos modos de hacer y producir periodismo que entraña. Pero al sobrevalorar la perspectiva de la producción podemos perder de vista justamente lo que lo hace particularmente valioso: cada pieza derivada del periodismo de datos suele exigir una intensiva participación del usuario que transforma el puzzle de data organizados por la agencia periodística en información con sentido.

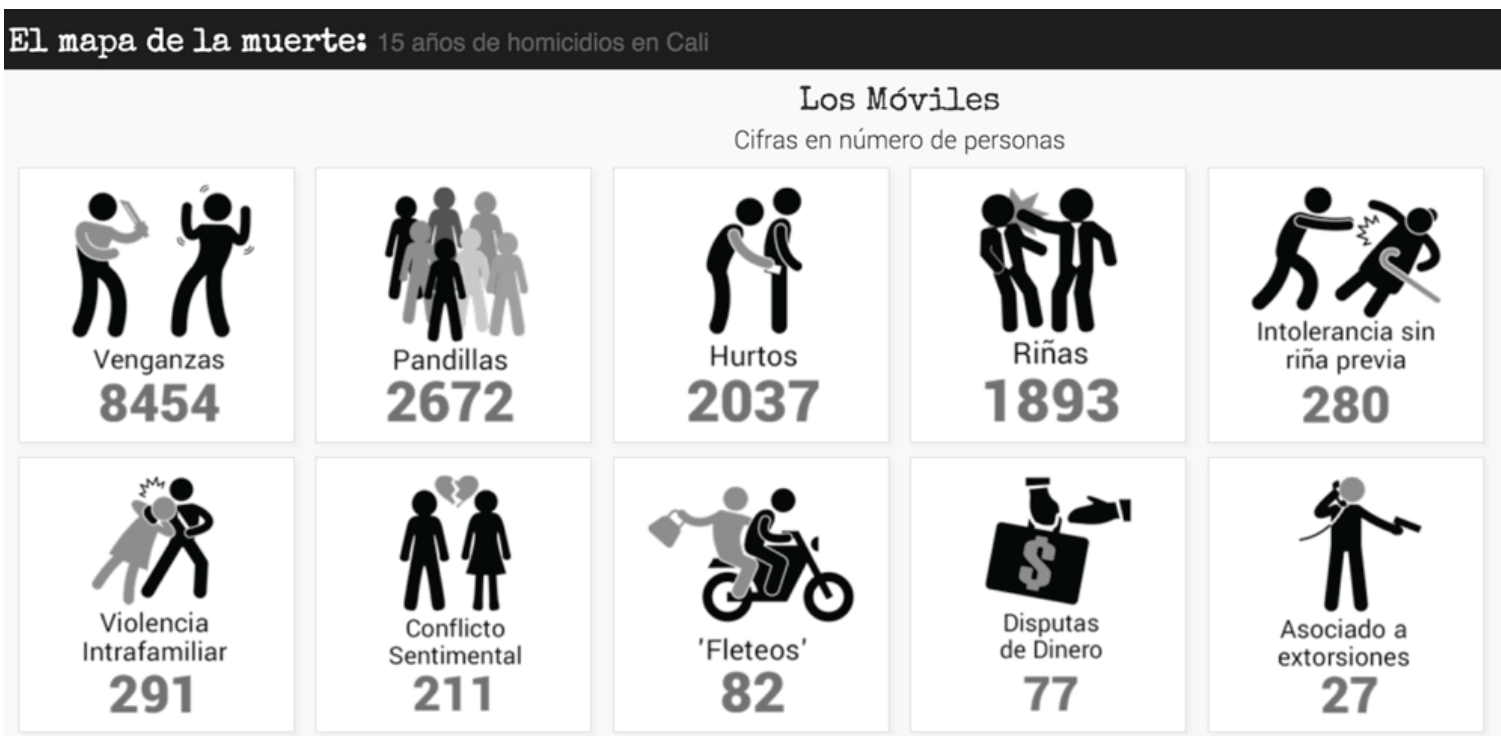

Figura 3. Proyecto El mapa de la muerte en Cali. http://www.elpais.com.co/especiales/el-mapa-de-la-muerte/ 


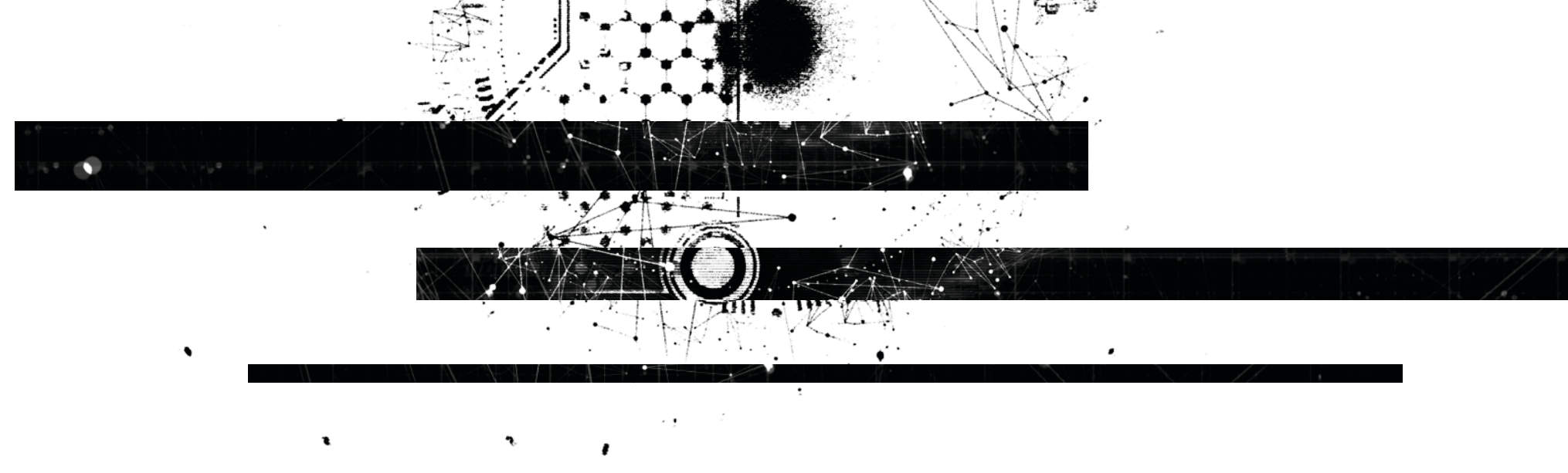

El agente que al final del proceso comunicacional le da sentido a los data organizados por el medio informativo puede ser otro medio de comunicación, un ciudadano común, una organización pública o privada que toma decisiones y modela acciones a partir de los data publicados. Quizás el rasgo más significativo del periodismo de datos es ese: procura datos organizados que demandan en los usuarios una lectura nueva y más exigente que las piezas periodísticas convencionales, pues la obra derivada del periodismo de datos se deja leer, principalmente, al manipularla. Como en los rompecabezas, no basta con reconocer sus fragmentos, es indispensable darles algún tipo de orden involucrándose intensivamente en ellos. De ahí que no dudemos en subrayar, respecto al periodismo de datos - tal como lo indicamos para el periodismo de análisis y algunas formas de periodismo de investigación- que estamos ante formas emergentes de periodismo postfactual o postinformativo, esto es, un tipo de práctica periodística en que los hechos ya no son el punto de llegada, ya no son el final de un proceso más o menos estandarizado de constatación, sino el punto de partida (González Mina, 2004).

\section{Resultados}

¿Cómo se organizan los data en el periodismo de datos? Por un lado, aún son usuales en algunas piezas el texto verbal (que cumple las funciones básicas de ciertos tipos discursivos: narración, argumentación, instrucción y descripción). Sin excepción las piezas analizadas suponen alguna puesta en relato de eventos y hechos significativos relacionados con las distintas formas de violencia y exterminio abusivo del otro. Pero, por otro lado, aparecen tanto recursos propios de los nuevos medios como recursos convencionales y típicos de la reportería clásica. Al lado de fotografías y videos, o viñetas y recuadros que compendian descripciones y relacionan datos acerca de los victimarios, las víctimas o los lugares y fechas en que ocurrieron los eventos, podemos apreciar mapas interactivos, infogramas, tablas, gráficos estadísticos dinámicos e infografías. Dos atributos caracterizan las formas de organización de información en las piezas estudiadas: las inferencias interpretativas y los vínculos interactivos más o menos automatizados entre data. En cierto sentido, se trata del mismo procedimiento sólo que en el primer caso hay un esfuerzo orientado a conectar y relacionar datos mediante argumentación verbal y yuxtaposición textual de los data, y en el segundo caso, de una articulación entre data mediante hipervínculos o automatización de bajo nivel (Manovich, 2005). 


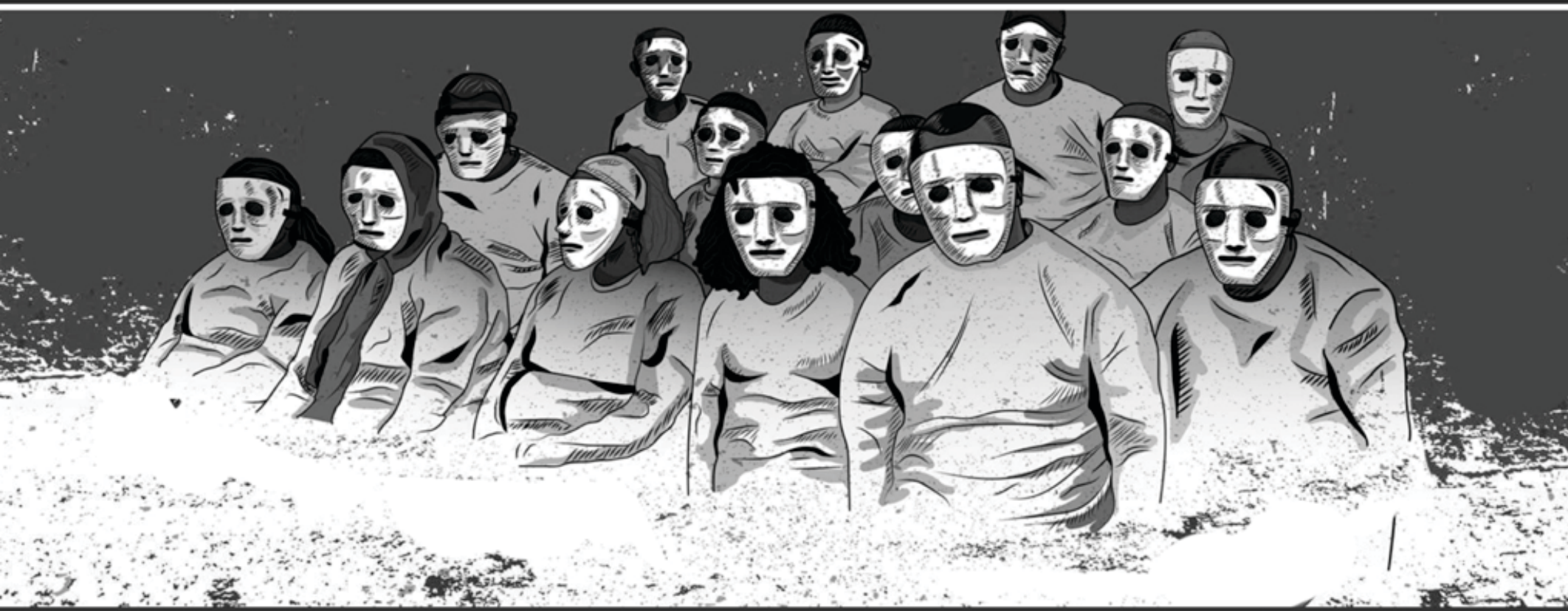

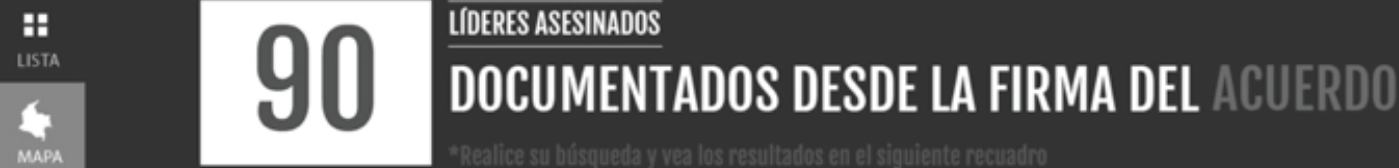

Figura 4. Proyecto La paz en el terreno. http://lapazenelterreno.com/lideres-sociales

¿De qué manera se configura esta información de tercer nivel a través de vínculos técnicos o vínculos inferenciales, esto es, usando la máquina o usando la palabra como recursos vinculares? La Figura 1, la más visible y generalizada, son los esfuerzos de geo y cronorreferenciación de eventos. Esto es, el uso de mapas dinámicos o estáticos en los que apreciar los eventos, las trayectorias, las rutas de los fenómenos. Las masacres cometidas por los paramilitares y su representación en mapas dinámicos en Rutas del conflicto: masacres ocurridas en Colombia desde el año 1982 hasta 2013, los mapas de desaparición forzada en México y Colombia en Desaparecidos Duelo Eterno, la geografía de los homicidios en Cali en El mapa de la muerte, o la geolocalización los asesinatos en la Paz en Terreno, y la persistente violencia en El Magdalena medio intenta cicatrizar sus heridas ${ }^{16}$, suponen la primera forma dominante de construcción de información que busca hacer comprensible las relaciones que entretejen los hechos.

La Figura 2 procura encontrar relaciones explicativas entre hechos se puede resumir en los siguientes términos: visualización de contrastes y comparaciones. Las piezas periodísticas examinadas ofrecen un conjunto de oportunidades para que los usuarios establezcan comparaciones entre fenómenos. Los recursos para poner en marcha tales comparaciones y contrastes son básicamente dos: las equivalencias en cifras y las correlaciones dinámicas gráficas entre fenómenos ${ }^{17}$. La Figura 3 de este tipo consiste en la representación de vínculos de poder mediante grafos o 
Por su condición estratégica también suele ser una noción abusada, es decir, usada en tantos y tan variados sentidos que termina por vaciarse de contenido hasta achatarse. Lo que llamamos interacciones máquina-agente humano son, en sentido estricto, inter(re)acciones. Esto que, en apariencia, parece un simple juego de palabras tiene consecuencias notables y claves para entender y avizorar el porvenir.

1. Lo interesante es que, como ocurre con los videojuegos o el click sobre un sitio web, la "interacción" comprometa al agente humano (persona) y al agente no humano (máquina) de manera fuertemente local, implicando -sólo de manera más bien periférica- el mundo off line de la persona.

2. La forma más intensa y profunda de interactividad (inter-re-actividad) máquina-agente humano es, sin duda, la del videojuego, pero algunas formas de periodismo de datos pueden estar incursionando - como en el video $360^{\circ}$ de El País- en esta frontera (El País, 2016).

3. Lo interesante es que el periodismo digital ha intentado, al pensarse en clave interactiva, imitar el concepto de "interactividad" que moviliza y opera el videojuego, o, al menos, la forma elemental de "interactividad" que se expresa en el clic. De hecho, desde el periodismo digital de inmersión hasta los gamejournalisme -que incluso ya tienen premios (gamesjournalismprize.com/) han llevado al extremo esta idea de inter(re)actividad.

4. Estas formas de inte(re)actividad necesariamente exploran sólo un modelo de "interactividad" que llamaremos aquí el modo de inter(re)actividad agente humano-no humano de alcance local. (Local refiere a la acción que ejerce el operador sobre la máquina y la eficacia de la máquina sobre quien opera; no refiere al alcance y cobertura telemática del mismo. Es decir, igual es local manipular -a distancia- un computador al otro lado del mundo).

5. Han emergido antes de la web y con la web otras formas de interactividad cuya relevancia reside no tanto en la arquitectura de las inter(re)acciones máquina-agente humano, sino en la profundidad de aquello que le hacen tales inter(re)acciones al mundo off line del agente humano. Esa inter(re) actividad que, por decirlo de un modo, agencia muchas más porciones de la vida off line del agente humano, mucho más que sus emociones localizadas, algo más que las pulsaciones de su corazón o el movimiento de sus dedos sobre el ratón o el teclado, encuentra en dos configuraciones sociotécnicas sus expresiones más decantadas: las redes sociales digitales y las aplicaciones para teléfonos móviles. Ambas implican un tipo nuevo de síntesis off line-on line, sin antecedentes en las formas locales de inter(re) actividad agente humano (persona)/agente no humano (computador)20.

6. Lo relevante de las redes sociales es el tipo de interactividad que, fundada en inter(re)actividades simples -subir una imagen, escribir un texto, mandar un mensaje, etc- compromete profundas acciones sobre la vida de las personas. La eficacia comunicativa de estas interacciones reside en que afectan la vida en general de las personas, tanto que no interesan realmente- las inter(re)acciones máquina-agente humano, sino la escala y dinamismo de las acciones mundo off line/on line. La interacción mundo off line - on line es mucho más relevante aquí que la inter(re)actividad 
técnica puramente operativa.

7. Las aplicaciones - para buscar taxi, para encontrar una potencial pareja en la ciudad que camino, para encontrar lugares donde comer, etc- hacen lo mismo que Facebook pero a la inversa: si en Facebook el mundo digital y on line ocupa el centro de la interacción, esto es, la porción significativa de la interacción se realiza en la plataforma Facebook; en las aplicaciones los términos se invierten, y las reglas del mundo off line, el entorno gravitacional que es la ciudad, la calle, el aeropuerto constituyen la plataforma real en que se despliega y opera la aplicación. Si no obtengo un taxi a tiempo, la aplicación no funciona. Los síntomas de ineficacia de las aplicaciones que operan síntesis online/offline se manifiestan como fracasos concretos en el mundo offline ${ }^{21}$.

8. Los medios de comunicación y, en particular, el periodismo cometen un error importante al sobrestimar la interactividad o inter(re)actividad local agente humano-máquina, y al subrayar aspectos ingenieriles y maquínicos, de programación, orientados a introducir mejoras para favorecer este tipo de inter(re)actividad. Aunque han explorado las redes sociales, en general parece que hay más bien descuidos, y se han usado como modos de promoción indirecta del medio, antes que verdadera fuente de interacciones con los usuarios; como modo otro de diseminación de información y promociones, y, en menor medida, como fuente de información. Y las aplicaciones han sido usadas como modos de distribución y anclaje en los teléfonos móviles de usuarios potenciales.

9. La débil exploración de la interactividad profunda off line/on line es, a nuestro juicio, un problema crucial para el desarrollo de medios de comunicación de nuevo y viejo tipo. La ruta explorada por los medios actuales, que se precian de interactivos, digitales, on line, subordinados al paradigma de la inter(re)actividad local humano/máquina de computar o cuerpo/pantalla - lo que ha supuesto una costosa apuesta en favor del diseño, la ingeniería y las diversas formas de puesta en escena digital- es significativamente errada.

10. Si uno quiere hacerse a una idea de lo que pueden llegar a ser formas de interactividad off line/on line para los medios de comunicación del futuro, hace falta echarle un vistazo breve al pasado: la forma perfecta de interactividad medio de comunicación-mundos de vida social se llamó periodismo ideológico o de agitación social o, en general, prensa popular asociada a movilizaciones sociales (González Mina, 2004). Es claro que este periodismo del des-orden social no le convenía a las formas instituidas de poder y dominación gubernamental y empresarial del capitalismo tardío y, en la actualidad, de las burocracias tecnocráticas. Por supuesto, las movilizaciones de ayer, hoy y el futuro, relacionadas con medios de comunicación, no se explican por la acción del periódico o de los medios, pero sí suelen obrar de manera recursiva y compleja con ellos. Pero imaginemos por un momento el tipo de exploraciones que, mediante esta interactividad profunda (offline/online), podrían desarrollarse a través de, por ejemplo, los periodismos on line: Escena 1. La inscripción a una red social ambientalista exige la aprobación de un tercero, previamente inscrito en la red. Ese tercero debe verificar que, efectivamente, usted haya 
realizado una acción pública y relevante de defensa ambiental: siembra efectiva de cinco árboles específicos en lugares definidos de la ciudad, un viaje de reconocimiento a Tenerife (Palmira, Colombia) para observar los nacimientos de agua y avistar los bosques de niebla y los extensos territorios de la palma de cera, o asistir a una manifestación pública contra la minería en los páramos; o, en una iniciativa en favor de la memoria de los conflictos sociales en Colombia, haya contribuido efectivamente al reconocimiento local y público de una víctima del conflicto armado.

Puede notarse cómo se trata de un tipo de "medio de comunicación" distinto. Uno puede imaginárselos como medios de comunicación cuyos contenidos, literalmente, se siembran en diversos lugares de la ciudad y en que los usuarios son intensamente co-creadores de los mismos porque implican una participación y compromiso activo muy fuerte. Asumir la interactividad profunda supone entender que el centro del medio no es el texto sino la creación de condiciones para que los "usuarios" creen y vivan contenidos y situaciones más o menos pre-diseñadas por los medios usando como plataforma de fondo no la web, en sí misma, sino en relación con la otra plataforma clave: la ciudad, los entornos off line.

11. En fin, al cambiar y pensar el paradigma de la interactividad como algo más que la inter(re)acción local cuerpo-agente humano-máquina-agente no humano, podemos salir de la trampa ingenieril que está desaprovechando lo más potente de las nuevas máquinas: la posibilidad de agenciar síntesis inéditas y configuraciones que reorganizan las fronteras mundo on line/off line. No hay que contentarse con las pequeñas interacciones locales, en tiempo real, que satisfacen el pequeño ideal de dominio y pseudoparticipación interactiva de los usuarios. El video 3600 del El País (2016) es, en ese sentido, un atisbo del porvenir.

El potencial de los nuevos medios reside menos en su arquitectura técnica, que en sus prometedoras derivas sociales, unas que recentran el papel de los usuarios en los procesos de construcción, reorganización y producción de sentido a partir de los recursos de información que las organizaciones informativas periodísticas siembran en sus entornos de vida. 


\section{Notas}

${ }^{1}$ Este artículo es derivado del proyecto de investigación Periodismo Emergente: Mutaciones, Técnicas y Estéticas, avalado por la Vicerrectoría de Investigaciones de la Universidad del Valle (Cód. 3203).

${ }^{2}$ Docente de la Universidad del Valle. Comunicador Social, Magister en Literaturas Colombiana y Latinoamericana.

${ }^{3}$ Docente de la Universidad del Valle, Comunicador Social, Doctor en Psicología.

${ }^{4}$ Consultar en: https://www.theguardian.com/media/2010/nov/22/data-analysis-tim-bernerslee

${ }^{5}$ A fundamental way newspaper sites need to change. Consultar en: http://www.holovaty.com/ writing/fundamental-change/

${ }^{6}$ Manual de periodismo de datos iberoamericano. http://manual.periodismodedatos.org/libro.php

${ }^{7}$ Consultar en: https://www.icij.org/

${ }^{8}$ Consultar en: https://www.icij.org/investigations/panama-papers/pages/panama-papers-aboutthe-investigation/

${ }^{9}$ Rutas del conflicto: masacres ocurridas en Colombia desde el año 1982 hasta 2013 http:// rutasdelconflicto.com/geografia-del-terror/masacres_desaparecidos.php

10 Consultar en: http://www.eltiempo.com/multimedia/especiales/desaparecidos-dueloeterno/16382245/1/index.html

${ }^{11}$ Consultar en: http://www.elpais.com.co/especiales/el-mapa-de-la-muerte/

${ }^{12}$ Consultar en: http: / / lapazenelterreno.com/lideres-sociales

${ }^{13}$ Consultar en: https://verdadabierta.com/especiales-v/2016/magdalena-medio/index.html

${ }^{14}$ Consultar en: http: / / lasillavacia.com/quienesquien

${ }^{15}$ Un ejemplo ilustrativo de datas que producen información en permanente y continuo cambio son las plataformas como http://www.worldometers.info/es/ o https://www.webpagefx. com/internet-real-time/.

${ }^{16}$ Consultar en: https://verdadabierta.com/especiales-v/2016/magdalena-medio/index.html

${ }^{17}$ Un ejemplo de este procedimiento es Desaparecidos: Duelo Eterno, que ofrece una visión panorámica y comparada del fenómeno en Colombia y México.

${ }^{18}$ Ver en El Mapa de la Muerte (El País, 2016), el apartado referido a Los Capos.

${ }^{19}$ De hecho, usualmente reservan un apartado para que los usuarios puedan incluir o aportar nuevos datos o ajustar los disponibles.

${ }^{20}$ Hay otros sectores de producción sociotécnica que están explorando poderosas formas de articulación de lo digital con entornos y operaciones no digitales off line: la realidad aumentada y los dispositivos de apoyo a la conducción de vehículos son dos ejemplos importantes de articulación digital/no digital, online/offline, muy poderosos.

${ }^{21}$ Por supuesto, nos referimos a esas aplicaciones que, en efecto, procuran soluciones y articulaciones fuertes con el mundo off line. (Excluimos de estas consideraciones aquellas aplicaciones enteramente relacionadas con entornos web y digitales). 


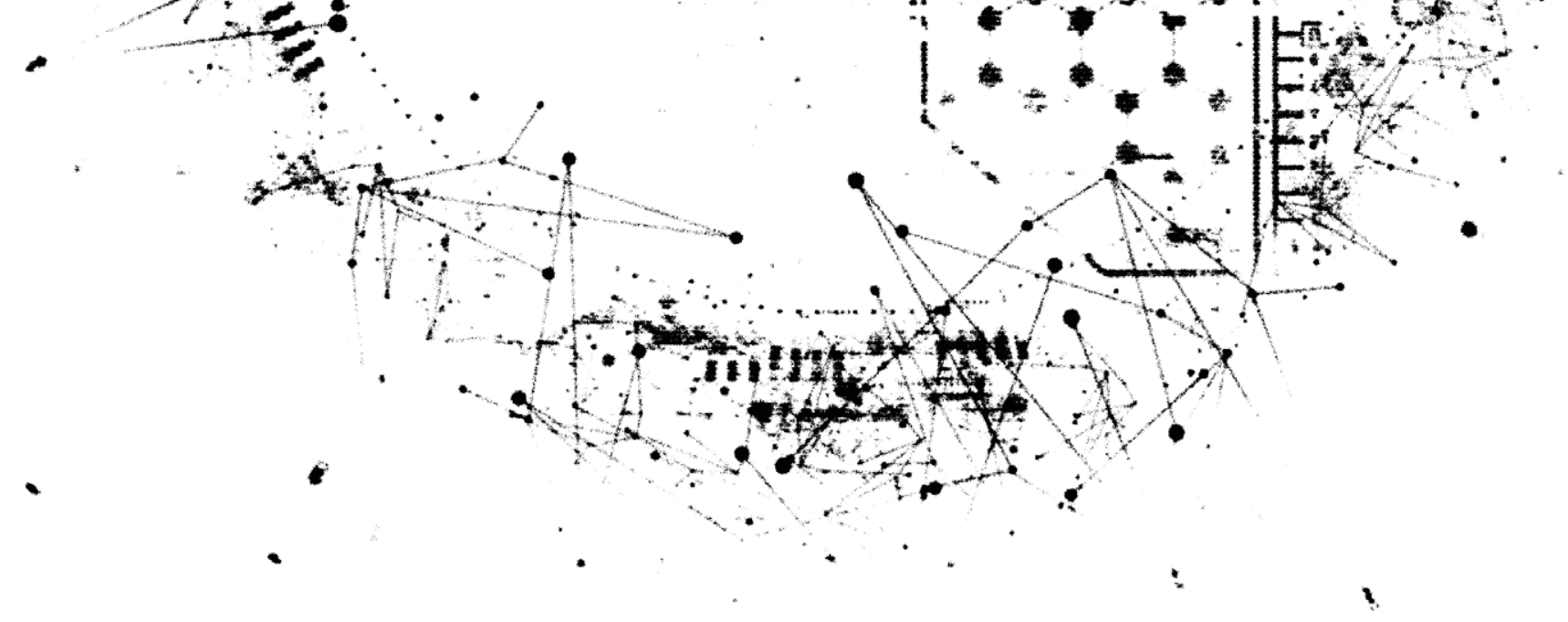

\section{Referencias}

Ávila, A. M. Manual de Periodismo de Datos Iberoamericano. Obtenido de http:/ / manual.periodismodedatos.org/ana-maria-avila.php

Comisión Nacional de Reparación y Reconciliación. Área de Memoria Histórica. (2013). ¡Basta ya! Colombia: Memorias de guerra y dignidad. Centro Nacional de Memoria Histórica.

Eco, U. (1992). Obra abierta. Barcelona: Planeta.

El País. (2016). El mapa de la muerte. Recuperado el 18 de Agosto de 2017, de sitio web del periódico El País, Cali: https://goo.gl/9YkqDq

El Tiempo. (Septiembre de 2015). Desaparecidos duelo eternoRecuperado el 7 de Diciembre de 2017, de Sitio web del periódico El Tiempo: https://www.eltiempo.com/multimedia/ especiales/desaparecidos-duelo-eterno/16382245/1/

Ferreras Rodríguez, E. M. (2013). Aproximación teórica al perfil profesional del Periodista de datos. Icono 14, 11(2), pp. 115-140. DOI: 10.7195/ri14.v11i2.573

Ferreras Rodríguez, E. (2016). El Periodismo de Datos en España. Estudios Sobre El Mensaje Periodístico, 22(1), 255-272. https: / / doi.org/10.5209/rev_ESMP.2016.v22.n1.52594

Flores, F. (1994). Creando organizaciones para el futuro. Santiago de Chile: Dolmen Ediciones.

Galindo, F. (2004): "Propuesta de periodización histórica y evolución conceptualdel periodismo de precisión”. Estudios sobre el Mensaje Periodístico, 10,Madrid, pp. 97-112

García, K. (12 de Marzo de 2016). Principios organizadores en seis medios emergentes en Internet. Presentación Resultados de Investigación. Cali: Escuela de Comunicación Social, disponible en https: / / goo.gl/hVbD3h

Gray, J., Bounegru, L., \& Chambers, L. (2012). Manual de periodismo de datos 1.0. Recuperado de http: / /interactivos.lanacion.com.ar/manual-data/

Gibson, J. J. (1972/2002). A Theory of DirectVisual Perception. En A. Noë, \& E. Thompson (Edits.), Vision and Mind. Selected Readings in the Philosophy of Perception. (págs. 77-89). Cambridge, Massachusetts: Massachusetts Institute of Technology.

González Mina, J. A. (2004). Repensar el periodismo: transformaciones y emergencias del periodismo actual. Cali: Programa Editorial, Universidad del Valle.

González, J. (2013). Niños que videojuegan. Videojuegos que estructuran tiempos. . Cali: Tesis Doctoral. Doctorado en Psicología. Universidad del Valle.

Holovaty, A. (2006). A fundamental way newspaper sites need to change. Recuperado de http://www. holovaty.com/writing/fundamental-change/

La Silla Vacía. (2016). Quién es quién. Recuperado el 15 de Enero de 2018, de sitio web de La Silla Vacía: https://goo.gl/hxwWXq

León, J. (spring-summer 2011). La Silla Vacía. Eight Ways the Internet Changes the Way We Think about the News. En ReVista. Harvard Review of Latin America. Recuperado de https: / / revista. drclas.harvard.edu/book/la-silla-vac\%C3\%ADa 
Manovich, L. (2005). El lenguaje de los nuevos medios de comunicación. La imagen en la era digital (Primera Edición en Inglés, 2001 ed.). (Ó. Fontrodona, Trad.) Barcelona: Ediciones Paidós.

Manovich, L. (2013). El software toma el mando (Vol. Comunicación No. 29). (Y. García Porres, Trad.) Barcelona: UOCpress.

Rutas del Conflicto, El Espectados, ICFJ, Friederich Ebert Stiftung. (s.f.). La paz en el terreno. Líderes sociales. Recuperado el 15 de Enero de 2018, de Sitio web La Paz en el Terreno: https:// goo.gl/pwR12g

Segnini, G. (2008). Introducción al Periodismo Investigativo y al Periodismo Asistido por Computadora. Ciudad de Guatemala:Agencia de los Estados Unidos para el Desarrollo Internacional.

Shannon, C. (July, October de 1948). A Mathematical Theory of Comunication. The Bell System Techical Journal, 27, 379-423.

The Guardian. (2010, noviembre 22). Analysing data is the future for journalists, says Tim Berners-Lee. Recuperado de https://www.theguardian.com/media/2010/nov/22/data-analysistim-berners-lee

Verdad Abierta. (2016). El Magdalena medio intenta cicatrizar sus heridas. Recuperado el 15 de Enero de 2018, de sito web de Verdad Abierta.com: https: / /goo.gl/TZuWeA

Verdad Abierta, ConLupa.co, Centro Nacional de Memoria Histórica. (s.f). Rutas del Conflicto. Cartografía del Conflicto. Recuperado el 15 de Diciembre de 2017, de Sitio web de Verdad Abierta: https://rutasdelconflicto.com/notas/mapa-interactivo-masacres-perpetradascolombia

Recibido: 4 de octubre de 2019 / Aprobado: 22 de diciembre de 2019 